\title{
Sélection séquentielle de conditions expérimentales non contrôlées
}

\author{
Luc Pronzato \\ Laboratoire I3S (CNRS - UNSA) \\ Les Algorithmes - Bât Euclide B \\ 2000 route des Lucioles - BP 121 \\ F-06903 Sophia Antipolis Cedex \\ pronzato@i3s.unice.fr
}

RÉSUMÉ. Nous considérons le problème posé par la sélection séquentielle de conditions expérimentales en vue de la construction d'un modèle paramétrique. La présentation se place à un niveau théorique (étude des propriétés asymptotiques d'une stratégie de sélection), mais plusieurs domaines d'application peuvent être envisagés (essais cliniques, modélisation de comportement par sondage d'opinion, physique expérimentale, fouille ou entreposage de données...). Dans tous les cas la «qualité » des données est quantifiée à partir d'un critère de planification d'expérience $\Phi$, fonction de la matrice d'information de Fisher. Nous donnons une règle de décision asymptotiquement optimale, qui conduit à une expérience $\Phi$-optimale respectant la contrainte que seulement une fraction $\alpha$ des données est sélectionnée, avec $\alpha$ fixé a priori.

ABSTRACT. We consider a sequential experimental design problem for the construction of a parametric model. The presentation is rather theoretical (we study the asymptotic properties of a selection rule), but several types of applications can be foreseen (clinical trials, survey sampling, questionnaire construction, experimental physics, data mining...). In all cases, the "quality" of the data is measured by an experimental design criterion, function of the Fisher information matrix. A decision rule is constructed that yields asymptotically a $\Phi$-optimum experiment under the constraint that only a fraction $\alpha$ of the data are selected, with $\alpha$ chosen a priori.

MOTS-CLÉS : planification d'expériences, planification séquentielle, échantillonnage, protocole expérimental, fouille de données, extraction d'information, entreposage de données.

KEYWORDS: experimental design, sequential design, sampling, data mining, extraction of information, data deposition.

RS - JESA. Volume 40 - n² 2/2006, pages 197 à 209 


\section{Introduction}

Nous souhaitons construire un modèle paramétrique, et, dans ce but, nous examinons séquentiellement des conditions expérimentales caractérisées par des grandeurs $X_{k} \in \mathcal{X} \subseteq \mathbb{R}^{q}, k=1,2, \ldots$, dont nous supposons qu'elles forment une suite de variables aléatoires indépendantes identiquement distribuées (avec une mesure de probabilité $\mu$ ). Dès qu'une nouvelle variable $X_{k}$ est examinée, nous devons décider « en ligne » si elle doit être acceptée ou pas.

Il peut s'agir de variables déjà présentes en masse dans une grande base de données, trop grande pour être utilisée dans sa totalité afin de construire le modèle qui nous intéresse : dans ce cas, nous tentons d'extraire séquentiellement de cette masse la partie la plus informative pour la construction du modèle (fouille de données). Il peut s'agir aussi de recueillir de nouvelles variables «dans la nature », et de faire vivre une base de données en ne lui incorporant que les grandeurs les plus informatives, toujours en termes de construction de modèle (entreposage de données). Dans d'autres situations, les variables $X_{k}$ peuvent correspondre à des caractéristiques immédiatement accessibles, par exemple sur des patients lors d'essais cliniques, ou à partir d'un questionnaire abrégé proposé à des individus interrogés pour un sondage d'opinion. Ces caractéristiques permettent de sélectionner directement et séquentiellement les situations les plus informatives pour la construction de modèle envisagée. Dans tous les cas, il s'agit d'être sélectif, et la démarche adoptée ici consiste à n'accepter qu'une fraction $\alpha$ des variables expérimentales $X_{k}$ proposées, $\alpha \in(0,1)$.

Comme de coutume, la qualité des conditions expérimentales est quantifiée par une fonction $\Phi$ de la matrice d'information de Fisher. On se place dans le cas où, pour des variables expérimentales $X_{1}, X_{2}, \ldots, X_{n}$, cette matrice est de la forme

$$
\mathbf{M}_{n}=\sum_{k=1}^{n} \mathbf{f}\left(X_{k}\right) \mathbf{f}^{\top}\left(X_{k}\right)
$$

où $\mathbf{f}(\cdot)$ dépend du modèle paramétrique considéré, $\operatorname{avec} \operatorname{dim}(\mathbf{f})=\operatorname{dim}(\theta)$ et $\theta$ les paramètres d'intérêt. Nous supposons que $\mathbf{f}(\cdot)$ est une fonction continue de $X$. Ceci recouvre aussi bien le cas de la modélisation de la probabilité de succès dans une loi de Bernoulli (modèle logistique par exemple), que celui plus classique d'un modèle de régression, linéaire ou non. Notons que si le modèle est non linéaire en ses paramètres $\theta, \mathbf{f}(\cdot)$ dépend de leur valeur. Nous supposons alors qu'une valeur nominale $\hat{\theta}^{0}$ est disponible, laquelle est utilisée pour calculer $\mathbf{f}(\cdot)$ (l'estimation récursive de $\theta$, et la mise à jour de $\mathbf{f}(\cdot)$ qui en découle, sont envisagées dans la section 4$)$.

Le critère $\Phi(\cdot)$ (à maximiser) est choisi concave, croissant et linéairement différentiable sur l'ensemble des matrices symétriques définies positives; $\Phi(\mathbf{M})=$ $\log \operatorname{det}(\mathbf{M})$ et $\Phi(\mathbf{M})=-\operatorname{trace}\left(\mathbf{M}^{-1}\right)$ sont des choix classiques, voir par exemple (Silvey, 1980). Nous notons $\mathcal{F}_{\Phi}\left(\mathbf{M}_{1}, \mathbf{M}_{2}\right)$ la dérivée directionnelle $\lim _{\epsilon \rightarrow 0^{+}}\{\Phi[(1-$ $\left.\left.\epsilon) \mathbf{M}_{1}+\epsilon \mathbf{M}_{2}\right]-\Phi\left(\mathbf{M}_{1}\right)\right\} / \epsilon$.

Soit $\left(u_{k}\right)_{k}$ la suite des décisions prises, avec $u_{k}=1$ si $X_{k}$ est accepté, $u_{k}=0$ sinon ( $X_{k}$ est connu quand la décision est prise). Si la suite des variables $X_{k}$ proposées 
est de longueur $N$ tandis que seulement un nombre $n<N$ peut en être accepté, le problème à résoudre correspond à maximiser

$$
\mathbb{E}\left\{\Phi\left(\mathbf{M}_{N, n} / n\right)\right\}
$$

par rapport à $\left(u_{k}\right)_{k}$ satisfaisant les contraintes

$$
u_{k} \in \mathcal{U}_{k} \subseteq\{0,1\}, k=1, \ldots, N, \sum_{k=1}^{N} u_{k}=n,
$$

avec

$$
\mathbf{M}_{N, n}=\sum_{k=1}^{N} u_{k} \mathbf{f}\left(X_{k}\right) \mathbf{f}^{\top}\left(X_{k}\right) .
$$

L'espérance mathématique $\mathbb{E}\{\cdot\}$ dans [1] porte sur $X_{1}, \ldots, X_{N}$ et concerne donc la mesure produit $\mu^{\otimes N}$. On peut donner de ce problème une formulation du type contrôle optimal stochastique.

A l'étape $k, 1 \leq k \leq N$, quand il s'agit d'accepter ou rejeter $X_{k}$, notons $a_{k}$ le nombre de $X_{i}$ déjà retenus :

$$
a_{k}=\sum_{i=1}^{k-1} u_{i}
$$

avec $a_{1}=0$. On peut alors considérer $\mathbf{s}_{k}=\left(a_{k}, \mathbf{M}_{k-1, a_{k}}, X_{k}\right)$ comme l'état et $u_{k}$ comme la commande du «système » au « temps » $k$. Une stratégie $S_{N, n}$ est définie par une fonction $(k, \mathbf{s}) \mapsto u \in\{0,1\}$ qui fournit la décision 0 ou 1 quand le système est dans l'état $\mathbf{s}$ à l'instant $k$. Pour tout $k \in\{1, \ldots, N\}$, la décision optimale est obtenue par la résolution du problème suivant :

$$
\begin{aligned}
& \max _{u_{k} \in \mathcal{U}_{k}} {\left[\mathbb { E } _ { X _ { k + 1 } } \left\{\operatorname { m a x } _ { u _ { k + 1 } \in \mathcal { U } _ { k + 1 } } \left[\mathbb { E } _ { X _ { k + 2 } } \left\{\max _{u_{k+2} \in \mathcal{U}_{k+1}}[\ldots\right.\right.\right.\right.} \\
&\left.\left.\left.\left.\left.\mathbb{E}_{X_{N-1}}\left\{\max _{u_{N-1} \in \mathcal{U}_{N-1}}\left[\mathbb{E}_{X_{N}}\left\{\max _{u_{N} \in \mathcal{U}_{N}}\left[\Phi\left(\sum_{i=1}^{N} u_{i} \mathbf{f}\left(X_{i}\right) \mathbf{f}^{\top}\left(X_{i}\right)\right)\right]\right\}\right]\right\} \ldots\right]\right\}\right]\right\}\right],
\end{aligned}
$$

où $\mathbb{E}_{X_{j}}\{$.$\} désigne l'espérance mathématique sur X_{j}$, qui est distribué avec la mesure de probabilité $\mu$, et

$$
\mathcal{U}_{j}=\mathcal{U}_{j}\left(a_{j}\right)=\left\{\begin{array}{cl}
\{0\} & \text { si } a_{j}=n \\
\{1\} & \text { si } a_{j}+N-j+1 \leq n \\
\{0,1\} & \text { sinon. }
\end{array}\right.
$$

Le cas $\operatorname{dim}(\theta)=1$ est considéré dans (Pronzato, 2001a) (le problème rejoint alors par certains aspects celui dit « de la secrétaire », voir par exemple (Freeman, 1983; Ferguson, 1989)). La stratégie optimale (en boucle fermée) est obtenue par programmation dynamique, et une règle de décision « boucle ouverte » est proposée, asymptotiquement optimale pour $n$ fixé et $N$ tendant vers l'infini (sa construction repose sur 
la distribution des valeurs extrêmes pour $\mu$, et l'optimalité asymptotique est obtenue pour une fonction de distribution des $X_{k}$ de type Von Mises).

La programmation dynamique ne conduit plus aussi simplement à la solution optimale dans le cas multidimensionnel $(\operatorname{dim}(\theta)>1)$. C'est ce cas qui va nous intéresser ici. Des stratégies sous-optimales sont proposées dans (Pronzato, 1999) (boucle ouverte avec retour d'information) et (Pronzato, 2001b) (« optimale à un pas », c'està-dire sur un horizon glissant de longueur 1). La première s'avère fortement sousoptimale.

La démarche que nous suivons ici repose sur la construction d'un protocole expérimental approximatif (approximative design). Soit $\Xi$ l'ensemble des mesures de probabilité sur $\mathcal{X}$. Pour tout $\xi \in \Xi$, nous notons

$$
\mathbf{M}(\xi)=\int_{\mathcal{X}} \mathbf{f}(x) \mathbf{f}^{\top}(x) \xi(d x)
$$

et $\phi(\xi)=\Phi[\mathbf{M}(\xi)]$. Nous supposons naturellement que $\mathbf{M}(\mu)$ existe et que $-\infty<$ $\phi(\mu)<\infty$, avec $\mu$ la mesure de probabilité pour chaque $X_{k}$. Nous supposons de plus que $\mu$ ne possède pas d'atomes, c'est-à-dire que pour tout ensemble $\Delta \mathcal{X}$ tel que $\mu(\Delta \mathcal{X})>0$ il existe $\Delta \mathcal{X}^{\prime} \subset \Delta \mathcal{X}$ tel que $0<\mu\left(\Delta \mathcal{X}^{\prime}\right)<\mu(\Delta \mathcal{X})$ (avec les mesures absolument continues par rapport à la mesure de Lebesgue comme cas particulier). Puisque $\Phi(\cdot)$ est linéairement différentiable, nous avons $F_{\Phi}\left(\xi_{1} ; \xi_{2}\right)=$ $\mathcal{F}_{\Phi}\left[\mathbf{M}\left(\xi_{1}\right), \mathbf{M}\left(\xi_{2}\right)\right]=\int_{\mathcal{X}} F_{\Phi}\left(\xi_{1}, x\right) \xi_{2}(d x)$ pour tout $\xi_{1}, \xi_{2}$ dans $\Xi$ avec $\phi\left(\xi_{1}\right)>-\infty$, où nous notons $F_{\Phi}(\xi, x)=F_{\Phi}\left(\xi ; \delta_{x}\right)$ et $\delta_{x}$ la mesure de Dirac en $x$. Par exemple, le cas de la $D$-optimalité où $\Phi(\cdot)=\log \operatorname{det}(\cdot)$ donne

$$
\begin{aligned}
F_{\Phi}(\xi, x) & =\lim _{\epsilon \rightarrow 0^{+}} \frac{1}{\epsilon}\left\{\log \operatorname{det}\left[(1-\epsilon) \mathbf{M}(\xi)+\epsilon \mathbf{M}\left(\delta_{x}\right)\right]-\log \operatorname{det} \mathbf{M}(\xi)\right\} \\
& =\frac{\partial \log \operatorname{det}\left[(1-\epsilon) \mathbf{M}(\xi)+\epsilon \mathbf{f}(x) \mathbf{f}^{\top}(x)\right]}{\partial \epsilon} \mid 0^{+} \\
& =\operatorname{trace}\left\{\mathbf{M}^{-1}(\xi)\left[\mathbf{f}(x) \mathbf{f}^{\top}(x)-\mathbf{M}(\xi)\right]\right\} \\
& =\mathbf{f}^{\top}(x) \mathbf{M}^{-1}(\xi) \mathbf{f}(x)-p,
\end{aligned}
$$

avec $p=\operatorname{dim}(\theta)$.

Nous ne considérons que le comportement asymptotique pour $n=\lfloor\alpha N\rfloor$, avec $\alpha \in(0,1)$ et $N \rightarrow \infty$. En suivant une démarche similaire à celle de Albright et Derman (1972), qui correspond au cas $\operatorname{dim}(\theta)=1$, nous montrons à la section 3 que la détermination d'une mesure $\xi_{\alpha}^{*}$ optimale pour $\phi(\cdot)$ sous la contrainte $\xi_{\alpha}^{*} \leq \mu / \alpha$ permet la construction d'une solution asymptotiquement optimale pour la maximisation de [1]. Les propriétés des mesures optimales satisfaisant des contraintes de ce type sont rappelées dans la section 2. Nous montrons ensuite (section 4) que la détermination de $\xi_{\alpha}^{*}$ n'est pas un préalable nécessaire, et qu'une stratégie asymptotiquement optimale permet en quelque sorte d'échantillonner asymptotiquement suivant $\xi_{\alpha}^{*}$ sans 
construire $\xi_{\alpha}^{*}$, ni même connaître $\mu$ à l'avance. Nous montrons enfin comment une simple adaptation de $\alpha$ redonne la stratégie «optimale à un pas » proposée dans (Pronzato, 2001b) quand $N$ et $n$ sont fixés. Un exemple illustratif est présenté à la section 5 . La section 6 dégage quelques perspectives à ce travail.

\section{Mesures bornées optimales}

Soit $\mathcal{D}(\mu, \alpha)$ l'ensemble des mesures de probabilité sur $\mathcal{X}$ satisfaisant la contrainte

$$
\xi(d x) \leq \mu(d x) / \alpha
$$

avec $\int_{\mathcal{X}} \xi(d x)=1$. Nous considérons dans cette section la construction d'une mesure $\xi_{\alpha}^{*} \in \mathcal{D}(\mu, \alpha)$ qui maximise $\phi(\xi)$ (mesure $\Phi$-optimale). Comme nous le verrons par la suite, il s'agit d'une étape importante vers la construction d'une stratégie asymptotiquement optimale pour la maximisation de [1].

Le théorème suivant est démontré dans (Wynn, 1982).

Théorème 1. Pour tout $\xi$ dans $\mathcal{D}(\mu, \alpha)$ il existe $\xi^{\prime}$ dans $\mathcal{D}^{*}(\mu, \alpha)$ telle que $\mathbf{M}(\xi)=$ $\mathbf{M}\left(\xi^{\prime}\right)$, avec

$$
\mathcal{D}^{*}(\mu, \alpha)=\{\xi \in \mathcal{D}(\mu, \alpha) / \exists \mathcal{A} \subset \mathcal{X}, \xi(\mathcal{A})=\mu(\mathcal{A}) / \alpha, \xi(\mathcal{X} \backslash \mathcal{A})=0\} .
$$

La concavité de $\Phi$ et la convexité de $\mathcal{D}(\mu, \alpha)$ impliquent l'existence d'une mesure $\Phi$-optimale $\xi_{\alpha}^{*}$ dans $\mathcal{D}(\mu, \alpha)$. (Notons que $\mathbf{M}\left(\xi_{\alpha}^{*}\right)$ est unique si $\Phi(\cdot)$ est strictement concave.) D'après le théorème précédent, $\xi_{\alpha}^{*} \in \mathcal{D}^{*}(\mu, \alpha)$. La différentiabilité de $\Phi$ permet de donner une condition nécessaire et suffisante d'optimalité pour $\xi_{\alpha}^{*}$, voir (Wynn, 1982; Fedorov, 1989; Fedorov et al., 1997; Sahm et al., 2001).

Théorème 2. Une condition nécessaire et suffisante pour que $\xi_{\alpha}^{*}$ maximise $\phi(\xi)$ sur $\mathcal{D}(\mu, \alpha)$ est qu'il existe une constante $c$ telle que $F_{\Phi}\left(\xi_{\alpha}^{*}, x\right) \geq c$ pour $\xi_{\alpha}^{*}$-presque tout $x$, et $F_{\Phi}\left(\xi_{\alpha}^{*}, x\right) \leq c$ pour $\left(\mu-\xi_{\alpha}^{*}\right)$-presque tout $x$.

Cette condition peut être reformulée en disant que la fonction $F_{\Phi}\left(\xi_{\alpha}^{*}, x\right)$ sépare l'ensemble

$$
\mathcal{X}_{\alpha}^{*}=\operatorname{supp} \xi_{\alpha}^{*}=\left\{x \in \mathcal{X} / \xi_{\alpha}^{*}(x)>0\right\}
$$

de son complémentaire $\mathcal{X} \backslash \mathcal{X}_{\alpha}^{*}$, voir (Wynn, 1982; Fedorov, 1989). On peut montrer également que $\int_{\mathcal{X}_{\alpha}^{*}} F_{\Phi}\left(\xi_{\alpha}^{*}, x\right) \mu(d x)=\int_{\mathcal{X}} F_{\Phi}\left(\xi_{\alpha}^{*}, x\right) \xi_{\alpha}^{*}(d x)=0$, voir (Fedorov, 1989; Fedorov et al., 1997). On notera $\phi_{\alpha}^{*}=\phi\left(\xi_{\alpha}^{*}\right)$, fonction continue décroissante de $\alpha$.

La situation est plus complexe lorsque $\mu$ possède des atomes, et on trouvera une extension du théorème précédent dans (Sahm et al., 2001) (qui traite aussi le cas de 
mesures bornées inférieurement). Voir également (Pronzato, 2004) pour une autre formulation de la condition nécessaire et suffisante du théorème 1. Des algorithmes itératifs (algorithmes «d'échange ») pour la construction de $\xi_{\alpha}^{*}$ sont présentés dans (Fedorov, 1989; Fedorov et al., 1997).

\section{Stratégie asymptotiquement optimale}

Pour toute stratégie $S_{N, n}$ utilisée pour la maximisation de [1], nous notons $\Psi\left(S_{N, n}\right)=\Phi\left(\mathbf{M}_{N, n} / n\right)$. Nous suivons la même démarche que (Albright $e t$ al., 1972), qui correspond au cas $\operatorname{dim}(\theta)=1$, et utilisons comme référence la stratégie qui consiste à attendre que les $N$ variables soient proposées pour en sélectionner $n$. Cette stratégie non-séquentielle, que nous notons $S_{N, n}^{*}$, correspond à la construction d'un protocole expérimental à $n$ points sur un ensemble fini de cardinal $N$. Pour tout $N$ et $n$, toute stratégie $S_{N, n}$, séquentielle ou pas, satisfait

$$
\Psi\left(S_{N, n}\right) \leq \Psi\left(S_{N, n}^{*}\right) .
$$

Par ailleurs, lorsque $N$ tend vers l'infini avec $n=\lfloor\alpha N\rfloor, \alpha \in(0,1)$, les performances de $S_{N, n}^{*}$ ne peuvent dépasser $\phi_{\alpha}^{*}$, c'est-à-dire

$$
\forall \alpha \in(0,1), \limsup _{N \rightarrow \infty} \Psi\left(S_{N,\lfloor\alpha N\rfloor}^{*}\right) \leq \phi\left(\xi_{\alpha}^{*}\right), \text { p.s. }(\mu),
$$

avec $\xi_{\alpha}^{*}$ une mesure bornée $\Phi$-optimale, $\xi_{\alpha}^{*} \leq \mu / \alpha$.

Considérons à présent la stratégie

$$
S_{N, n}^{\epsilon}:\left\{\begin{array}{l}
\text { accepter } X_{k} \text { si } X_{k} \in \mathcal{X}_{\alpha+\epsilon}^{*}, \text { ou si } N-k+1 \leq n-a_{k} \\
\text { rejeter } X_{k} \text { sinon, }
\end{array}\right.
$$

avec $0 \leq \epsilon<1-\alpha, a_{k}$ donné par [2] et $\mathcal{X}_{\alpha+\epsilon}^{*}$ par [4]. Nous la simplifions encore en considérant $\bar{S}_{N, n}^{\epsilon}$ définie par

$$
\bar{S}_{N, n}^{\epsilon}:\left\{\begin{array}{l}
\text { accepter } X_{k} \text { si } X_{k} \in \mathcal{X}_{\alpha+\epsilon}^{*} \\
\text { rejeter } X_{k} \text { sinon. }
\end{array}\right.
$$

$\bar{S}_{N, n}^{\epsilon}$ peut conduire à accepter moins de $n$ points, et donc, puisque $\Phi(\cdot)$ est croissant, $\Psi\left(S_{N, n}^{\epsilon}\right) \geq \Psi\left(\bar{S}_{N, n}^{\epsilon}\right)$. Notons $N_{\alpha+\epsilon}$ le nombre de variables $X_{k}$ qui auraient été acceptées si la contrainte $a_{k} \leq n$ était ignorée. Il satisfait $N_{\alpha+\epsilon} / N \rightarrow \alpha+\epsilon$, p.s. $(\mu)$ quand $N$ tend vers l'infini. Par conséquent, pour $n=\lfloor\alpha N\rfloor$, $\operatorname{Prob}\left\{N_{\alpha+\epsilon} \leq\right.$ $n$ infiniment souvent $\} \rightarrow 0$, et asymptotiquement $\bar{S}_{N, n}^{\epsilon}$ échantillonne $n=\lfloor\alpha N\rfloor$ fois suivant $\xi_{\alpha+\epsilon}^{*}$. Ceci donne

$$
\Psi\left(\bar{S}_{N,\lfloor\alpha N\rfloor}^{\epsilon}\right) \rightarrow \phi_{\alpha+\epsilon}^{*}, \text { p.s. }(\mu)
$$

et donc

$$
\liminf _{N \rightarrow \infty} \Psi\left(S_{N,\lfloor\alpha N\rfloor}^{\epsilon}\right) \geq \phi_{\alpha+\epsilon}^{*}, \text { p.s. }(\mu)
$$


Il suffit à présent de faire tendre $\epsilon$ vers zéro, et la continuité en $\alpha$ de $\phi_{\alpha}^{*}$, voir (Pronzato, 2004), donne le résultat suivant à partir de [5,6] et [8].

Théorème 3. La stratégie $S_{N, n}^{0}$ définie par [7] est asymptotiquement optimale pour $n=\lfloor\alpha N\rfloor, N \rightarrow \infty, 0<\alpha<1$ :

$$
\lim _{N \rightarrow \infty} \Psi\left(S_{N,\lfloor\alpha N\rfloor}^{0}\right)=\lim _{N \rightarrow \infty} \Psi\left(S_{N,\lfloor\alpha N\rfloor}^{*}\right)=\phi\left(\xi_{\alpha}^{*}\right), \text { p.s. }(\mu)
$$

avec $\xi_{\alpha}^{*}$ une mesure bornée $\Phi$-optimale $\left(\xi_{\alpha}^{*} \leq \mu / \alpha\right)$ et $S_{N, n}^{*}$ la stratégie non séquentielle qui consiste à sélectionner $n$ variables parmi $N$ connues.

L'utilisation de $S_{N, n}^{0}$ nécessite la construction préalable de $\mathcal{X}_{\alpha}^{*}$, c'est-à-dire de la mesure bornée $\Phi$-optimale $\xi_{\alpha}^{*}$. Nous allons voir à présent qu'il est cependant possible d'éviter cette construction.

\section{Tirages asymptotiques suivant une mesure bornée optimale}

D'après le théorème 2, et puisque $\operatorname{Prob}\left(X_{1} \in \mathcal{X}_{\alpha}^{*}\right)=\mu\left(\mathcal{X}_{\alpha}^{*}\right)=\alpha$, la stratégie $S_{N, n}^{0}$ est encore définie par

$$
S_{N, n}^{0}:\left\{\begin{array}{l}
\text { accepter } X_{k} \operatorname{si} \mu\left\{x / F_{\Phi}\left(\xi_{\alpha}^{*}, x\right)>F_{\Phi}\left(\xi_{\alpha}^{*}, X_{k}\right)\right\}<\alpha \\
\text { ou si } N-k+1 \leq n-a_{k}, \\
\text { rejeter } X_{k} \text { sinon }
\end{array}\right.
$$

Afin de s'affranchir de la connaissance de la mesure $\xi_{\alpha}^{*}$, nous la remplaçons simplement par la mesure empirique des $X_{k}$ déjà sélectionnés, que nous notons $\xi_{k}$. Nous définissons ainsi une nouvelle stratégie $S_{\alpha}(\mu)$.

$$
S_{\alpha}(\mu): \begin{cases}\text { accepter } X_{k} \text { si } P_{k} & =P_{k}\left(X_{k}\right) \\ & =\mu\left\{x / F_{\Phi}\left(\xi_{k}, x\right)>F_{\Phi}\left(\xi_{k}, X_{k}\right)\right\}<\alpha \\ \text { rejeter } X_{k} \text { sinon } & \end{cases}
$$

Lorsque $X_{k}$ est accepté, $a_{k+1}=1+a_{k}$ et $\xi_{k+1}=\left[1-1 /\left(1+a_{k}\right)\right] \xi_{k}+1 /\left(1+a_{k}\right) \delta_{X_{k}}$, dans le cas contraire $a_{k+1}=a_{k}$ et $\xi_{k+1}=\xi_{k}$.

La procédure doit être initialisée par une matrice $\mathbf{M}$ non singulière. Une façon de procéder consiste à accepter les premiers échantillons proposés jusqu'à avoir $\mathbf{M}\left(\xi_{k}\right)$ de rang plein. Cette initialisation n'a bien entendu aucun effet sur le comportement asymptotique de la stratégie, que nous étudions à présent.

Deux raisons font que la suite $\left[\phi\left(\xi_{k}\right)\right]_{k}$ n'est pas monotone croissante : (i) la « longueur du pas »1/(1+ak $)$ est prédéterminée et (ii) $X_{k}$ est une variable aléatoire. Tandis que le point (i) est plutôt classique pour les algorithmes de construction d'expériences optimales et a été largement étudié (voir en particulier (Wu et al., 1978)), le point (ii) forme une originalité du problème considéré ici. 
Nous utilisons les deux hypothèses techniques suivantes. Elles permettent d'établir le théorème ?? dont nous ne donnons qu'une esquisse de démonstration. On pourra se référer à (Pronzato, 2003) pour la démonstration complète.

H1 : La dérivée seconde $\nabla_{\Phi}^{2}\left(\mathbf{M}_{1}, \mathbf{M}_{2}\right)$, définie par

$$
\nabla_{\Phi}^{2}\left(\mathbf{M}_{1}, \mathbf{M}_{2}\right)=\frac{\partial^{2} \Phi}{\partial \gamma^{2}}\left[(1-\gamma) \mathbf{M}_{1}+\gamma \mathbf{M}_{2}\right]_{\mid \gamma=0^{+}} .
$$

est continue en $\mathbf{M}_{1}$ et $\mathbf{M}_{2}$ pour toute matrice $\mathbf{M}_{1}$ telle que $\left|\Phi\left(\mathbf{M}_{1}\right)\right|<\infty$. Elle satisfait

$$
\int_{\mathcal{A}} \nabla_{\Phi}^{2}\left[\mathbf{M}_{1}, \mathbf{f}(x) \mathbf{f}^{\top}(x)\right] \mu(d x)>\Delta_{\alpha}(A)>-\infty
$$

pour tout ensemble $\mathcal{A}$ tel que $\mu(\mathcal{A}) \geq \alpha$ et toute matrice $\mathbf{M}_{1}$ telle que $\Phi\left(\mathbf{M}_{1} / 2\right)>A$, avec $\Delta_{\alpha}(A)$ une constante (qui dépend de $\alpha$ et $A$ ).

H2 : $\exists A>-\infty$ et $\epsilon>0$ tels que $\phi(\xi) \geq A$ pour toute mesure $\xi \in \mathcal{D}^{*}(\mu, \alpha-\epsilon)$, avec $\mathcal{D}^{*}$ défini par [3].

L'hypothèse $\mathrm{H} 1$ est satisfaite pour les critères classiques de planification, par exemple lorsque $\mathcal{X}$ est borné ou lorsque les composantes de $\mathbf{f}(x)$ sont des polynômes en $x$ et $\mu$ possède une densité (par rapport à la mesure de Lebesgue) qui décroît de façon exponentielle quand $x \rightarrow \infty$. H2 est satisfaite par exemple si les vecteurs $\mathbf{f}(x)$ sont indépendants sur tout sous-ensemble $\mathcal{A}$ de $\mathcal{X}$ de mesure $\mu(\mathcal{A})>\alpha-\epsilon$ et $\mu$ a une densité $\varphi(\cdot)$ satisfaisant $\varphi(x) \geq q>0$ sur $\mathcal{X}$. On pourra noter que c'est l'hypothèse $\mathrm{H} 2$ qui permet de s'affranchir du problème de la décroissance éventuelle de $\phi_{k}$ vers $-\infty$ que l'on peut rencontrer pour un algorithme de « longueur de pas » prédéterminée (voir le Dichotomous Theorem de Wu et Wynn (1978)).

Théorème 4. La mesure empirique $\xi_{k}$ générée par les points acceptés par la stratégie $S_{\alpha}(\mu)$ définie par [9] satisfait

$$
\lim _{k \rightarrow \infty} \phi\left(\xi_{k}\right)=\phi\left(\xi_{\alpha}^{*}\right), \text { p.s. }(\mu),
$$

avec $\xi_{\alpha}^{*}$ une mesure bornée $\Phi$-optimale, $\xi_{\alpha}^{*} \leq \mu / \alpha$.

DÉMONSTRATION.- On note $\phi_{k}=\phi\left(\xi_{k}\right)$. La première étape de la démonstration consiste à montrer que $\limsup _{k \rightarrow \infty} \phi_{k}=\phi_{\alpha}^{*}$ p.s. $(\mu)$ dès lors qu'existe $A>-\infty$ tel que $\liminf \inf _{k \rightarrow \infty} \phi_{k} \geq A$ (l'hypothèse $\mathrm{H} 2$ garantit l'existence p.s.( $\mu$ ) d'un tel $A$ ). Pour cela, on examine une itération lorsque $X_{k}$ est accepté, événement noté $A_{k}$ qui est de probabilité $\alpha$. Nous avons :

$$
\phi_{k+1}=\phi_{k}+\frac{1}{1+a_{k}} F_{\Phi}\left(\xi_{k}, X_{k}\right)+\frac{1}{2\left(1+a_{k}\right)^{2}} \nabla_{\Phi}^{2}\left(\xi_{k}, X_{k}, \gamma\right)
$$

avec

$$
\nabla_{\Phi}^{2}\left(\xi_{k}, X_{k}, \gamma\right)=\nabla_{\Phi}^{2}\left[(1-\gamma) \mathbf{M}\left(\xi_{k}\right)+\gamma \mathbf{f}\left(X_{k}\right) \mathbf{f}^{\top}\left(X_{k}\right), \mathbf{f}\left(X_{k}\right) \mathbf{f}^{\top}\left(X_{k}\right)\right]
$$


pour une valeur $\gamma \in\left[0,1 /\left(1+a_{k}\right)\right]$. La concavité de $\Phi(\cdot)$ implique $F_{\Phi}\left(\xi_{k} ; \xi_{\alpha}^{*}\right) \geq$ $\phi_{\alpha}^{*}-\phi_{k}$, c'est-à-dire

$$
\int_{\mathcal{X}} F_{\Phi}\left(\xi_{k}, x\right) \xi_{\alpha}^{*}(d x) \geq \phi_{\alpha}^{*}-\phi_{k}
$$

ou encore, puisque $\xi_{\alpha}^{*}=\mu / \alpha$ sur $\mathcal{X}_{\alpha}^{*}$ et vaut zéro sur $\mathcal{X} \backslash \mathcal{X}_{\alpha}^{*}$,

$$
\int_{\mathcal{X}_{\alpha}^{*}} F_{\Phi}\left(\xi_{k}, x\right) \mu(d x) / \alpha \geq \phi_{\alpha}^{*}-\phi_{k}
$$

On obtient donc

$$
\begin{aligned}
\mathbb{E}_{X_{k}}\left\{F_{\Phi}\left(\xi_{k}, X_{k}\right) \mid A_{k}\right\}= & \int_{\left\{x / P_{k}(x)<\alpha\right\}} F_{\Phi}\left(\xi_{k}, x\right) \mu(d x) / \alpha \\
& \geq \int_{\mathcal{X}_{\alpha}^{*}} F_{\Phi}\left(\xi_{k}, x\right) \mu(d x) / \alpha \geq \phi_{\alpha}^{*}-\phi_{k}
\end{aligned}
$$

On utilise à présent $\mathrm{H} 1$, qui conduit à

$$
\mathbb{E}_{X_{k}}\left\{\phi_{k+1} \mid A_{k}\right\}>\phi_{k}+\frac{1}{1+a_{k}}\left(\phi_{\alpha}^{*}-\phi_{k}\right)+\frac{\Delta_{\alpha}(A)}{2 \alpha\left(1+a_{k}\right)^{2}} .
$$

Lorsque $X_{k}$ est rejeté (événement de probabilité $1-\alpha$ ), $\phi_{k+1}=\phi_{k}$. En combinant cela avec [11], nous obtenons

$$
\mathbb{E}_{X_{k}}\left\{\phi_{k+1}-\phi_{k}\right\}>\frac{\alpha}{1+a_{k}}\left(\phi_{\alpha}^{*}-\phi_{k}\right)+\frac{\Delta_{\alpha}(A)}{2\left(1+a_{k}\right)^{2}},
$$

ce qui implique $\lim \sup _{k \rightarrow \infty} \phi_{k} \geq \phi_{\alpha}^{*}$ p.s. $(\mu)$, et donc $\lim \sup _{k \rightarrow \infty} \phi_{k}=\phi_{\alpha}^{*}$ p.s. $(\mu)$ d'après le théorème 3 .

La deuxième étape, plus technique, consiste à montrer que l'on ne peut pas avoir

$$
\limsup _{k \rightarrow \infty} \phi_{k}-\liminf _{k \rightarrow \infty} \phi_{k}>\epsilon>0 .
$$

On utilise pour cela une construction similaire à celle de la démonstration du Lemme de Doob, voir (Williams, 1991) page 106.

Revenons à présent au problème initial, avec $n$ variables à sélectionner dans une suite de longueur $N$. Une modification élémentaire de la stratégie $S_{\alpha}(\mu)$ consiste à adapter la constante $\alpha$ en la remplaçant par

$$
\alpha_{k}=\left(n-a_{k}\right) /(N-k)
$$

lorsqu'il s'agit de sélectionner (ou pas) $X_{k}$. Cette stratégie $S_{\alpha_{k}}(\mu)$ coïncide avec celle proposée initialement dans (Pronzato, 2001b). D'après les théorèmes 3 et 4 , elle est asymptotiquement optimale pour $n=\lfloor\alpha N\rfloor, \alpha \in(0,1)$, et $N \rightarrow \infty$. 
Lorsque la mesure $\mu$ est inconnue, ce qui est la situation la plus fréquente, la probabilité $P_{k}$ dans [9] peut être remplacée par une évaluation $\hat{P}_{k}$ obtenue à partir de la mesure empirique des $X_{k}$, soit $\hat{\mu}_{k}$ à l'itération $k$ (après avoir observé $X_{k}$ ). Puisque les $X_{i}$ sont observés indépendamment de la stratégie de sélection adoptée (ce qui correspond à une propriété de « séparation » en contrôle), le théorème 4 demeure valide pour la stratégie correspondante $S_{\alpha}\left(\hat{\mu}_{k}\right)$ (ou $S_{\alpha_{k}}\left(\hat{\mu}_{k}\right)$ ). On peut donc effectuer, de manière asymptotique, des tirages suivant $\xi_{\alpha}^{*}$ sans véritablement construire $\xi_{\alpha}^{*}$ ni même connaître $\mu$ à l'avance. Nous en présentons un exemple à la section suivante.

Notons enfin que si $\mathbf{f}(x)$ dépend des paramètres $\theta$ (modèle non linéaire), il est parfois possible d'estimer $\theta$ « en ligne », c'est-à-dire récursivement au fur et à mesure du recueil des observations. Considérons par exemple le cas de la régression non linéaire, avec $\hat{\theta}^{k}$ l'estimateur des moindres carrés obtenu à partir des $X_{i}$ déjà sélectionnés. Sous des hypothèses de régularité du modèle, l'hypothèse $\mathrm{H} 2$ garantit assez facilement la convergence et la normalité asymptotique de $\hat{\theta}^{k}$ (voir par exemple (Jennrich, 1969)). Dans le calcul de $P_{k}$, ou de $\hat{P}_{k}$, la valeur inconnue de $\theta$ peut alors être remplacée par $\hat{\theta}^{k}$, et la stratégie $S_{\alpha}(\mu)$ assure que $\phi_{k}$ converge p.s. $(\mu)$ vers $\phi\left[\xi_{\alpha}^{*}(\bar{\theta})\right]$, avec $\xi_{\alpha}^{*}(\bar{\theta}) \leq \mu / \alpha$ une mesure bornée $\Phi$-optimale pour la vraie valeur $\bar{\theta}$ des paramètres du modèle.

\section{Exemple}

L'exemple choisi est suffisamment simple pour que l'on puisse construire la mesure bornée $\Phi$-optimale $\xi_{\alpha}^{*}$. Les conditions expérimentales $X_{k}$ (scalaires) sont utilisées pour construire un modèle de régression quadratique $\eta(\theta, x)=\theta_{0}+\theta_{1} x+\theta_{2} x^{2}$ à partir d'observations $y_{k}=\eta\left(\bar{\theta}, X_{k}\right)+\epsilon_{k}$, avec $\bar{\theta}$ la vraie valeur (inconnue) des paramètres du modèle et $\left(\epsilon_{k}\right)_{k}$ une suite de variables aléatoires indépendantes et identiquement distribuées, de moyenne nulle et de variance $\sigma^{2}$. Les variables $\epsilon_{k}$ et $X_{k}$ sont indépendantes. Le critère de planification choisi est celui de $D$-optimalité, $\Phi(\cdot)=\log \operatorname{det}(\cdot)$. Les vecteurs $\mathbf{f}\left(X_{i}\right)$ correspondent aux régresseurs $\left(1, X_{i}, X_{i}^{2}\right)^{\top}$.

Supposons que les $X_{k}$ soient distribués suivant la loi normale $\mathcal{N}(0,1)$. Nous choisissons $\alpha=0.5$. On peut alors montrer que la mesure $\Phi$-optimale $\xi_{\alpha}^{*}$ qui satisfait $\xi_{\alpha}^{*} \leq \mu / \alpha$ coïncide avec $\mu / \alpha$ sur $\mathcal{X}_{\alpha}^{*}=(-\infty,-a] \cup[-b, b] \cup[a, \infty)$, avec $a \simeq 1.028$, $b \simeq 0.2482$. La figure 1 présente $\mathbf{f}^{\top}(x) \mathbf{M}^{-1}\left(\xi_{\alpha}^{*}\right) \mathbf{f}(x)=F_{\Phi}\left(\xi_{\alpha}^{*}, x\right)+3$ en fonction de $x$ et illustre le théorème 2 . La ligne en pointillés correspond à la constante $c$ du théorème.

La figure 2 présente un histogramme des 10000 premiers échantillons $X_{k}$ sélectionnés par la stratégie $S_{\alpha}\left(\hat{\mu}_{k}\right)$. La figure 3 présente $\phi_{k}$ en fonction de $k$, la valeur $\phi\left(\xi_{\alpha}^{*}\right)$ est indiquée par la ligne en pointillés. 


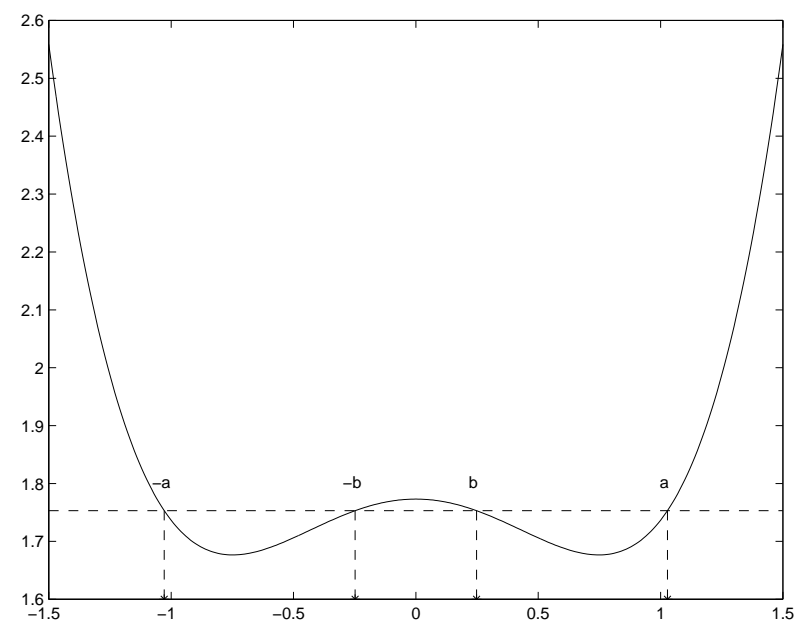

Figure 1. $\mathbf{f}^{\top}(x) \mathbf{M}^{-1}\left(\xi_{\alpha}^{*}\right) \mathbf{f}(x)$ en fonction de $x$

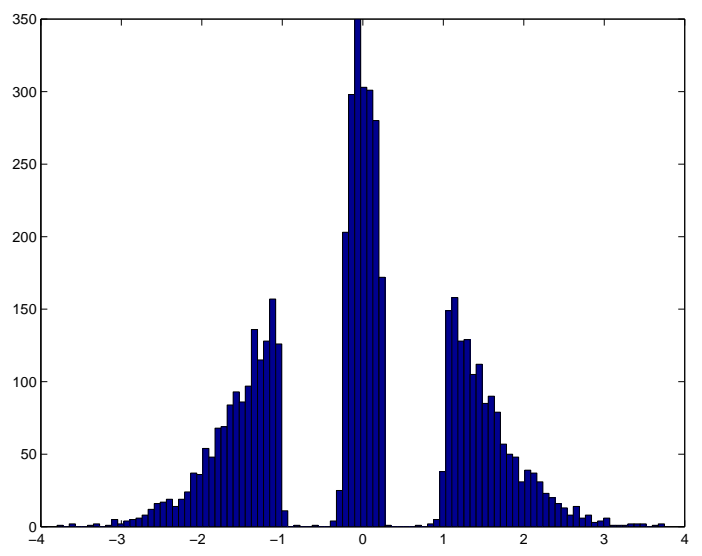

Figure 2. Histogramme des 10000 premiers échantillons $X_{k}$ sélectionnés par $S_{\alpha}\left(\hat{\mu}_{k}\right)$

\section{Conclusion et perspectives}

Une stratégie a été proposée pour sélectionner séquentiellement des conditions expérimentales. Elle est extrêmement simple à mettre en œuvre, ce qui permet d'envisager son utilisation même si les données sont en très grand nombre et les modèles à identifier de structure complexe. Elle est asymptotiquement optimale lorsqu'il s'agit de sélectionner une fraction $\alpha$ des données disponibles. 
L'étude des propriétés de cette stratégie lorsque le nombre $n$ de données à sélectionner est fixe tandis que la longueur $N$ de la séquence proposée tend vers l'infini reste à faire. Il est raisonnable de penser que les résultats obtenus en dimension 1 (Pronzato, 2001a) doivent pouvoir s'étendre au cas $\operatorname{dim}(\theta)>1$. Tous les cas intermédiaires pour lesquels $n$ tend vers l'infini avec $N$ mais $n / N \rightarrow 0$ seraient également à étudier.

Nous avons supposé ici que la mesure $\mu$ qui génère les $X_{k}$ ne possédait pas d'atomes. Le cas de mesures présentant des composantes discrètes, plus technique, est présenté dans (Pronzato, 2006). Enfin, reste à traiter le cas où les $X_{k}$ ne sont plus indépendants (systèmes dynamiques) et celui où l'on n'observe pas directement $X_{k}$ mais une covariable $Z_{k}$ telle que la loi conditionnelle $\pi(X \mid Z)$ soit connue. Une application importante pour ce deuxième cas concerne les essais cliniques : la sélection des patients à inclure dans un test pourrait alors se faire sur la base de covariables facilement accessibles (âge, taille, poids, etc.) tandis que les variables expérimentales entrant dans la modélisation (par exemple pharmacocinétique ou pharmacodynamique) ne seraient prises en compte que lors des expérimentations sur les individus sélectionnés.

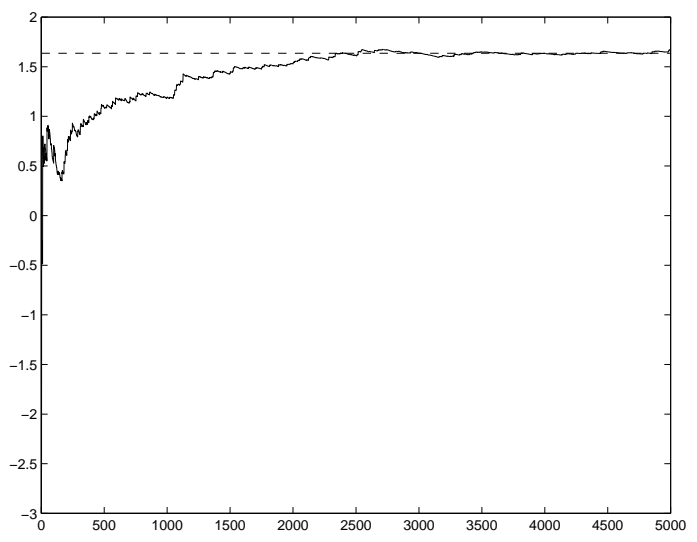

Figure 3. $\phi_{k}$ générée par $S_{\alpha}\left(\hat{\mu}_{k}\right)$ en fonction de $k$; la ligne en pointillés correspond $\grave{a} \phi\left(\xi_{\alpha}^{*}\right)$

\section{Remerciements}

Ce travail trouve son origine dans un commentaire de Valerii V. Fedorov (GlaxoSmithKline) lors d'un colloque sur la planification d'expériences qui s'est tenu à Cardiff en avril 2000, voir (Pronzato, 2001b); je le remercie pour avoir attiré mon attention sur les mesures bornées optimales. Diverses discussions avec Henry P. Wynn (London School of Economics) et Éric Thierry (Laboratoire I3S Sophia Antipolis) ont également été fort utiles. 


\section{Bibliographie}

Albright S., Derman C., "Asymptotically optimal policies for the stochastic sequential assignment problem", Management Science, vol. 19, n 1, 1972, p. 46-51.

Fedorov V., "Optimal design with bounded density : optimization algorithms of the exchange type”, Journal Statist. Planning and Inference, vol. 22, 1989, p. 1-13.

Fedorov V., Hackl P., Model-Oriented Design of Experiments, Springer, Berlin, 1997.

Ferguson T., "Who solved the secretary problem?", Statistical Science, vol. 4, n 3, 1989, p. 282-296.

Freeman P., "The secretary problem and its extensions : a review", International Statistical Review, vol. 51, 1983, p. 189-206.

Jennrich R., "Asymptotic properties of nonlinear least squares estimation", Annals of Math. Stat., vol. 40, 1969, p. 633-643.

Pronzato L., "Sequential selection of observations in randomly generated experiments", Tatra Mountains Mathematical Publications, vol. 17, 1999, p. 167-175.

Pronzato L., "Optimal and asymptotically optimal decision rules for sequential screening and resource allocation”, IEEE Transactions on Automatic Control, vol. 46, n 5, 2001a, p. 687 697.

Pronzato L., "Sequential construction of an experiment design from and i.i.d. sequence of experiments without replacement", in A. Atkinson, B. Bogacka, A. Zhigljavsky (eds), Optimum Design 2000, Kluwer, Dordrecht, chapter 11, 2001b, p. 113-122.

Pronzato L., "Designing from a sequence of i.i.d. experiments", Tatra Mountains Mathematical Publications, vol. 26, 2003, p. 11-28.

Pronzato L., "A minimax equivalence theorem for optimum bounded design measures", Statistics \& Probability Letters, vol. 68, 2004, p. 325-331.

Pronzato L., "On the sequential construction of optimum bounded designs", Journal of Statistical Planning and Inference, vol. 136, 2006, p. 2783-2804.

Sahm M., Schwabe R., "A note on optimal bounded designs", in A. Atkinson, B. Bogacka, A. Zhigljavsky (eds), Optimum Design 2000, Kluwer, Dordrecht, chapter 13, 2001, p. 131140.

Silvey S., Optimal Design, Chapman \& Hall, London, 1980.

Williams D., Probability with Martingales, Cambridge University Press, Cambridge, 1991.

Wu C., Wynn H., "The convergence of general step-length algorithms for regular optimum design criteria", Annals of Statistics, vol. 6, n 6, 1978, p. 1273-1285.

Wynn H., "Optimum submeasures with applications to finite population sampling", in S. Gupta, J. Berger (eds), Statistical Decision Theory and Related Topics III. Proc. 3rd Purdue Symp., vol. 2, Academic Press, New York, 1982, p. 485-495.

Reçu le : 25 juin 2005

Accepté le : 12 mars 2006 